\title{
Atrofia cortical posterior
}

\author{
Carolina D elgado D, Archibaldo D onoso S. \\ Posterior cortical atrophy. \\ Report of five cases
}

Posterior cortical atrophy (PCA) is a neurodegenerative syndrome, usually due to Alzheimer's disease. The first symptoms are progressive impairment of visuospatial (Balint's and Gertsmann's syndromes) or visuoperceptive (visual agnosia, alexia) function. Episodic memory and executive function are spared until later stages. We report two males aged 51 and 55 years and three females aged 50, 54 and 56 years, with posterior cortical atrophy. Ophthalmological study was normal in all. Presenting signs and symptoms were visual ataxia, simultagnosia, agraphia, acalculia, spatial disorientation and unilateral neglect (Balint's and Gerstmann's syndromes). Apperceptive visual agnosia, aphasia, apraxia and alexia were also observed. One female had cortical blindness. Structural images were inconclusive, but PET scan and SPECT disclosed functional impairments in occipitotemporal or occipito parietal areas (Rev Méd Chile 2009; 137: 1482-7).

(Key w ords: Alzheimer disease; Brain diseases; Posterior cortical atrophy)

Recibido el 19 de marzo, 2009. Aceptado el 16 de septiembre, 2009.

Hospital Clínico de la Universidad de Chile. Santiago de Chile.

L a atrofia cortical posterior (ACP) es un cuadro neurodegenerativo que compromete las funciones visuales superiores con respeto total 0 parcial de la vía genículo calcarina ${ }^{1,2}$. El primer caso fue publicado por Arnold Pick en 19021,3, el segundo, con agnosia visual asociativa, por Taylor y Warrington en 1972 1 ; pero su conocimiento más amplio sólo se inició en 1988, con la serie de Benson ${ }^{4}$. La mayoría de los pacientes consulta inicialmente a un oftalmólogo y la derivación a neurología es más tardía ${ }^{5}$. Al comienzo la memoria es normal o casi normal y no existe demencia; cuando existen olvidos precoces, éstos son rápidamente sobrepasados por los defectos visuoespaciales de mayor pregnancia ${ }^{6}$. Se han propuesto los siguientes criterios diagnósticos ${ }^{5}$ :

Correspondencia a: Dr. Archibaldo Donoso S. Las Malvas 539, Las Condes. E mail: adonoso@redclinicauchile.cl
1. Comienzo insidioso con síntomas de falla visual. 2. Ausencia de una patología oftalmológica que explique los síntomas; la agudeza visual es normal, puede existir restricción u otros defectos del campo visual. 3. Los defectos visuales son desproporcionados con respecto a otras fallas cognitivas. 4. Curso progresivo. 5. Las imágenes pueden mostrar atrofia, hipometabolismo o hipoperfusión occípito-parietal u occípito-temporal uni o bilaterales.

Clínicamente Benson supuso que podían ser variantes de la enfermedad de Alzheimer (EA) o de la enfermedad de Pick ${ }^{4}$. En 1999 Alberca señalaba que entre los 14 casos de ACP con más de 2 años de evolución (para descartar la forma posterior de la enfermedad de Creuztfeldt Jakob) que habían llegado a la autopsia, 13 correspondían a $\mathrm{EA}^{1}$. En la gran mayoría de los casos la autopsia ha mostrado una EA con placas seniles y degeneración neurofibrilar en áreas parieto-occi- 
pitales $^{2,5}$ y ha sido incluida entre las EA atípicas ${ }^{7,8}$. Tang-Wai destacó que las diferencias entre EA y ACP dependían más de la cuantía de la degeneración neurofibrilar en las áreas de Brodmann 17 y 18 y en parietal inferior que de las placas seniles; señaló que el respeto del hipocampo explicaría la conservación de la memoria episódica ${ }^{5}$. En casos aislados la autopsia ha mostrado una demencia mixta (EA con demencia por cuerpos de Lewy o enfermedad de Parkinson), gliosis subcortical, degeneración corticobasal, enfermedad de Creutzfeldt-Jakob, insomnio familiar fatal $2,5,6,9$. No se han descrito casos con patología de parálisis supranuclear progresiva ni demencia frontotemporal. La mayoría son mujeres y la edad de inicio es menor que en la EA clásica1,5,6. Casi todos los casos han sido esporádicos; algunos (como nuestro caso 5) tenían familiares con demencias tardías, que no correspondían a $\mathrm{ACP}^{6}$. La única excepción son las 2 hermanas publicadas por Otsuki, con un cuadro clínico característico pero tardío ${ }^{10}$.

Desde 1973 a la fecha hemos diagnosticado 5 casos, que corresponderían a 1-2\% de nuestros pacientes con EA. Presentaremos en detalle al último; los otros, incluyendo 2 ya publicados ${ }^{11} \mathrm{se}$ presentarán en forma resumida. Nuestro interés en comunicarlos reside especialmente en lo peculiar de las manifestaciones clínicas, que al no ser reconocidas retrasan el diagnóstico.

Presentación de los casos (Tabla 1): Caso 5. Hombre de 55 años, diestro, con 15 años de escolaridad, microempresario, previamente sano, cuya madre sufrió de una probable EA de inicio tardío. Su enfermedad se inició en forma insidiosa en 2003, con dificultades visuales; varios oftalmólogos encontraron agudeza visual normal y campimetría discretamente disminuida. Leía en forma muy lenta "le costaba juntar las letras" y tuvo que dejar de manejar por la dificultad en percibir los otros vehículos. Le era difícil encontrar un objeto, ya fuera un tenedor en la mesa del comedor o la manilla de una puerta. Caminaba en forma insegura y su esposa dijo "para ver los obstáculos debe dirigir la vista".

Fue evaluado a fines de 2007. En los últimos 8 meses se habían agregado leves fallas de memoria reciente, elementos depresivos y ansiedad. El examen médico general y el examen neurológico básico fueron normales; el campo visual por confrontación fue normal. A pesar de la agudeza visual normal, al pedirle contar dedos cometía errores, por ejemplo decía "2 ó 3" al mostrarle 5; a veces corregía su error. Estaba orientado, con noción de enfermedad y colaboraba bien al examen. La atención estaba conservada, repitió hasta 6 cifras en forma directa e invirtió bien los meses del año. El lenguaje oral conversacional era normal, con comprensión normal salvo en estructuras gramaticales pasivas o pasivo-negativas. Fue capaz de interpretar algunos proverbios en forma abstracta. No existían apraxia ideatoria, ideomotora, del vestir, oral ni del habla, pero sí una apraxia constructiva con descuido de detalles.

Denominó bien colores y objetos, pero con algunos dibujos cometió errores por percepción de detalles aislados: ante el dibujo de un barco dijo "¿una nota musical?” señalando una ventana ovalada; por una mesa dijo "unos pilares" refiriéndose a las patas del mueble. El pareo visual (de figuras muy disímiles entre sí) fue normal. Reconoció $6 / 8$ fotos de personajes famosos, fallando en evocar el nombre de 2 de ellos. Al describir la lámina 1 del test de Boston se refirió a algunos elementos de la escena con omisión de otros igualmente importantes y sin llegar a una síntesis global de la escena. Al pedirle contar varios elementos idénticos (varias letras 0 ) cometió errores por simultagnosia. Además, cuando debía revisar una serie de figuras en una tarea de asociación semántica, su rendimiento era lento y difícil, para explorar bien tomaba la lámina y la movía frente a sus ojos en diferentes direcciones (el rendimiento semántico fue bueno). El seguimiento visual era adecuado si el movimiento del blanco era lento, pero fallaba si se movía con rapidez. Reconocía bien la pantomima si el gesto era estático pero cometía errores si era dinámico. No existía ataxia óptica de la mano (con un blanco inmóvil) y el nistagmo optokinético horizontal y vertical estaba conservado.

Leía bien palabras aisladas y oraciones simples, a veces letra por letra. En párrafos largos se hacían evidentes sus defectos: omitía palabras, saltaba de un renglón a otro, cometía errores en palabras polisilábicas. Escribía con mala alineación de los renglones, algunos errores ortográficos y ocasional mezcla de letras (manuscritas con imprenta 0 mayúsculas). El cálculo era muy deficiente: hizo 
Tabla 1. Presentación de los 5 casos de ACP

\begin{tabular}{|c|c|c|c|c|c|}
\hline & 1 & 2 & 3 & 4 & 5 \\
\hline Sexo & M & $\mathrm{F}$ & $\mathrm{F}$ & $\mathrm{F}$ & M \\
\hline Edad inicio & 53 & 54 & 50 & 56 & 51 \\
\hline Edad examen & 55 & 57 & 56 & 63 & 55 \\
\hline Sobrevida & 7 años & $>12$ & $>8$ & 10 años & $>5$ \\
\hline Escolaridad (años) & 16 & 12 & 12 & 12 & 15 \\
\hline Síntomas iniciales & $\begin{array}{l}\text { ataxia óptica } \\
\text { desorientación } \\
\text { espacial }\end{array}$ & ataxia óptica & $\begin{array}{l}\text { agrafia } \\
\text { ataxia óptica }\end{array}$ & ataxia óptica & $\begin{array}{l}\text { alexia } \\
\text { ataxia óptica }\end{array}$ \\
\hline Olvidos & Sí & $\mathrm{Sí}^{\prime}$ & Sí & Sí & No \\
\hline Afectividad & Apatía & Anosodiaforia & $\begin{array}{l}\text { Depresión } \\
\text { psicótica }\end{array}$ & Apatía & $\begin{array}{l}\text { Ansiedad- } \\
\text { anosodiaforia }\end{array}$ \\
\hline Paramimesis & Sí & Sí & Sí & Sí & No \\
\hline Parkinsonismo & No & No & No & Simétrico & No \\
\hline Mioclonías o crisis TC & No & Sí & No & Sí & No \\
\hline Estudio oftalmológico & $\begin{array}{l}\text { Restricción } \\
\text { del CV }\end{array}$ & Normal & Normal & $\begin{array}{l}\text { Normal } \\
\text { ceguera cortical }\end{array}$ & Normal \\
\hline Demencia & Moderada & Moderada & Severa & Severa & Leve \\
\hline MM Folstein & - & $13 / 30$ & $5 / 30$ & $0 / 30$ & $22 / 30$ \\
\hline MPC Raven & - & No rinde & $5 / 36$ & No rinde & $7 / 36$ \\
\hline $\begin{array}{l}\text { Ataxia óptica } \\
\text { (con blanco fijo) }\end{array}$ & Sí & Sí & Sí $i>d$ & No rinde & No \\
\hline Seguimiento visual & Lento, falla rápido & Menor hacia izq. & No sigue & No sigue & Lento, falla rápido \\
\hline Negligencia unilateral & No & Izquierda & Izquierda & No & No \\
\hline Agnosia visual aperceptiva & Severa & Severa & Severa & Ceguera cortical & Leve \\
\hline Simultagnosia & Sí ++ & Sí ++ & No precisada & Ceguera cortical & $\mathrm{Si}+$ \\
\hline Prosopagnosia & Sí & Sí & No precisada & Ceguera cortical & No \\
\hline Alexia verbal & Severa & Moderada & Severa & Ceguera cortical & Leve \\
\hline Afasia & Amnésica & No & Amnésica & Global-mutismo & No \\
\hline Agrafia & Apráxica & Apráxica y espacial & Apráxica & No rinde & Espacial, leve \\
\hline Apraxias & $\begin{array}{l}\text { Ideatoria, del vestir, } \\
\text { constructiva }\end{array}$ & $\begin{array}{l}\text { Constructiva, } \\
\text { del vestir }\end{array}$ & $\begin{array}{l}\text { Constructiva, } \\
\text { del vestir }\end{array}$ & $\begin{array}{l}\text { No rinde } \\
\text { No rinde }\end{array}$ & Constructiva \\
\hline Acalculia & Espacial & Global & Global & No rinde & Global \\
\hline $\begin{array}{l}\text { Desorientación } \\
\text { der-izq, agnosia digital }\end{array}$ & Ambas & Ambas & No precisado & No rinde & Ambas \\
\hline Amnesia verbal & Moderada & Leve & Moderada & Severa & No \\
\hline FAB & - & - & $5 / 18$ & & $11 / 18$ \\
\hline EEG & Theta & Theta & Theta & & \\
\hline $\begin{array}{l}\text { Imagen estructural: } \\
\text { atrofia }\end{array}$ & $\begin{array}{l}\text { Neumo- } \\
\text { encefalografía: } \\
\text { difusa leve }\end{array}$ & RM: difusa leve & TAC: difusa leve & TAC: difusa severa & RM: normal \\
\hline $\begin{array}{l}\text { Imagen funcional: } \\
\text { hipofunción }\end{array}$ & & $\begin{array}{l}\text { SPECT: } \\
\text { TPO bilateral }\end{array}$ & & & PET: TO bilateral \\
\hline
\end{tabular}

bien algunas sumas simples, pero fracasó en la mayoría de las restas, cálculo escrito y la solución de un problema. Además existían desorientación derecha-izquierda y agnosia digital visual.

El compromiso de la memoria verbal era escaso, en aprendizaje verbal, llegó a 9/10 palabras en 10 ensayos; a los 10 minutos evocó 9 y reconoció 10/ 10. En memoria semántica evocó 12 nombres de animales en 60 segundos (normal >15); y evocó 7 de los últimos 8 presidentes de Chile.

En Minimental test de Folstein obtuvo 22/30 puntos, fallando especialmente en atención y cálculo (0/5 puntos) y en dibujo (copió sólo uno de los 2 pentágonos y no fue capaz de detectar su error). En Matrices Progresivas Coloreadas (MPC) obtuvo sólo 7/36 puntos. En la prueba FAB (Frontal Assessment 
Battery) obtuvo 11/18 puntos, fallando en secuencias motoras y en control inhibitorio.

En resumen, presentaba defectos en la exploración visual, simultagnosia, síndrome de Gertsmann, apraxia constructiva y agnosia visual aperceptiva, con respeto de memoria episódica, atención, lenguaje oral y función ejecutiva. La resonancia magnética cerebral fue informada como normal, y clínicamente se formuló el diagnóstico de ACP. Un PET scan en enero de 2008 mostró hipometabolismo occípito temporal bilateral (Figura 1).

En la Tabla 1 se presentan los datos de los 5 casos. Se pueden observar varios datos de interés: - Inicio precoz, entre 50 y 56 años (promedio 52,8).

- Diagnóstico tardío, con un promedio de 4,4 años de evolución. Antes del diagnóstico de ACP se habían formulado varios diagnósticos imprecisos: "ambliopía psicogénica" en el caso 1, "inatención visual" en el 5 y sólo "demencia" en el 4 . En este último caso no se dispuso de evaluaciones neuropsicológicas previas, pero hay elementos de juicio para diagnosticar una ACP. Sufrió de severos defectos visuales durante más de un año antes de que aparecieran las primeras fallas de memoria (era profesora, hacía clases pero colocaba las notas en el casillero de otro alumno; le costaba encontrar los objetos que estaban en su cómoda; chocó por no calcular la distancia con otros vehículos). Además llegó a la ceguera cortical en una etapa en que todavía conservaba la marcha (se había descartado toda patología ocular), lo que no hemos observado nunca en EA.

- La ataxia óptica fue un síntoma inicial importante. Se expresaba como dificultad para tomar un objeto, escribir en un teclado, o percibir los obstáculos durante la marcha. En la evaluación se corroboró la severa ataxia óptica al pedirles alcanzar un blanco fijo, sólo el caso 5 rindió bien en esta prueba, pero tenía fallas en su vida diaria.

- Los estudios oftalmológicos siempre fueron negativos.

- En todos (salvo el 4 que tenía una ceguera cortical) existía agnosia visual aperceptiva. En 2 de 3 casos se agregó prosopagnosia. La severidad del defecto visual hizo que su rendimiento en MPC fuera al azar.

- Todos (salvo el caso 4) tenían alexia de severidad variable, reconocían letras aisladas o podían leer palabras o frases simples. Esta alexia se asociaba a simultagnosia (dificultad

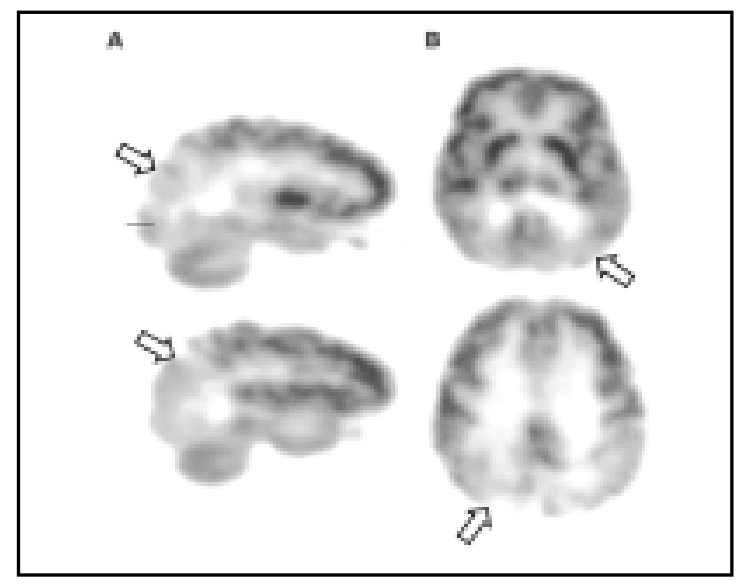

Figura 1. PET scan del caso 5. Las flechas señalan las áreas de hipometabolismo.

para contar varios elementos idénticos dispuestos en el papel).

- El seguimiento visual de un objeto en movimiento fue deficiente o imposible en todos. Este defecto, junto a la simultagnosia y la ataxia óptica, configuraba un síndrome de Balint.

- En la mayoría estuvo presente el síndrome de Gerstmann; en el caso 3 no se buscó y en el 4 era imposible. Las agrafias fueron de tipo apráxico (asociadas a apraxia constructiva), salvo en el caso 5, en que existían un desorden espacial y errores alográficos.

- Con respecto al lenguaje oral, el caso 4 casi no hablaba y se diagnosticó como afasia global con mutismo; en los otros el lenguaje oral era normal o existía sólo una afasia amnésica.

- En casi todos se agregó una demencia con alteraciones de la afectividad, fallas de atención, memoria y abstracción. En el caso 5 estos defectos eran mínimos, pero existían elementos del síndrome de Gertsmann y apraxia constructiva. Mostraba ansiedad frente a sus limitaciones.

- Los casos 2 y 4 presentaron mioclonías y luego crisis convulsivas generalizadas; en el 4 se agregaron parkinsonismo severo y disquinesias orofaciales (había recibido risperidona por agitación, pero después de instalado el parkinsonismo).

- Los exámenes estructurales cerebrales fluctuaron entre lo normal (caso 5) y una gran atrofia difusa (caso 4); en ninguno se hizo referencia a una atrofia de predominio posterior. Sin embargo, fueron exámenes disímiles; incluso 
en el primer caso fue una neumoencefalografía; y no se contó con ellos para que un radiólogo los estudiara en forma retrospectiva. En cambio, los exámenes funcionales, realizados en sólo dos casos, mostraron compromiso bilateral de las áreas de asociación PO o PTO.

\section{DiscUSIÓN}

Nuestros casos (salvo el paciente 4 que llegó en una etapa muy avanzada pero cuya historia era concordante) cumplen los requisitos necesarios para diagnosticar una ACP. Destacan la edad de inicio (promedio 52,8 años) y la pregnancia de los defectos visuales frente al compromiso menor y más tardío de memoria, lenguaje y otras funciones. Los trastomos neuropsicológicos de la percepción visual en la ACP pueden ser clasificados en dorsales y ventrales ${ }^{5,12}$. Los dorsales interrumpen las vías occípito-parietales e interfieren con la localización de lo percibido y el control visual del movimiento; se expresan como simultagnosia, ataxia óptica y parálisis psíquica de la mirada, o sea un síndrome de Balint. Este compromiso parietal también puede expresarse como un síndrome de Gertsmann, con desorientación derecha-izquienda, agnosia digital, agrafia y acalculia. Los trastomos de la pencepción visual de tipo ventral intemumpen las vías occípito-temporales, interfieren con la percepción o reconocimiento de los objetos y se expresan en defectos tales como la alexia sin agrafia, agnosia visual y prosopagnosia ${ }^{13,14}$. Las agnosias visuales generalmente han sido de tipo aperceptivo ${ }^{6}$.

Algunos casos de ACP comespondeńan a compromiso de las vías occípito-temporales, como el caso de Beversdorf y Heilmann ${ }^{14}$ en que existía alexia (incluso para la notación musical), prosopagnosia, agnosia y anomia visual. En otros casos predominan los problemas visuoespaciales por disfunción parietal, con síndromes de Balint y Gerstmann ${ }^{5,6,15}$. En los nuestros, como en muchos de los publicados, existe una mezcla de defectos de tipo dorsal y ventral ${ }^{4-6,16-}$ 18. Pueden llegar a la ceguera cortical, como sucedió en nuestro caso $4^{6}$. Varios pacientes publicados tuvieron alucinaciones y delinios de reconocimiento (síndrome de Capgras u otros) 6 .

Las alexias y agrafias están entre los signos más frecuentes de la $\mathrm{ACP}^{1,3,6}$. Con respecto a las alexias, Méndez observó que la dificultad para reconocer las letras aumenta cuando están entre otras parecidas; que se leían mejor las palabras reales que los logotomas; y que la estrategia de leer las letras una por una no era eficaz. Usó el término "alexia aperceptiva" a semejanza de la agnosia visual $^{3}$. McMonagle hizo hincapié en la importancia de la simultagnosia en la alexia ${ }^{6}$, lo que concuerda con nuestra impresión clínica. Las agrafias pueden ser lingüísticas, visuoespaciales y apráxicas; en nuestros casos predominó el último componente.

El cuadro clínico puede ser asimétrico, y la negligencia unilateral, la extinción visual e incluso la hemianopsia es mencionada por varios autores ${ }^{4,6,16}$. Tal como en nuestros casos 2 y 3, estos defectos comprometen más el hemicampo izquierdo. La variabilidad del compromiso cerebral también se expresa en otros signos: las afasias con parafasias fonémicas sugieren compromiso de la circunvolución supramarginal izquierda; una apraxia ideomotora apunta a la corteza parietal izquierda ${ }^{13}$. Nuestros casos -en la medida que pudieron ser evaluados- presentaron apraxias constructivas y del vestir; no tuvimos casos de apraxia ideomotora, y sólo uno de apraxia ideatoria, con menos valor localizador.

Las discretas alteraciones del tono muscular, los reflejos palmomentonianos, los defectos de memoria y los defectos del lenguaje eran compatibles con una enfermedad de Alzheimer; en los casos 2 y 4 se agregaron mioclonías y convulsiones tónico clónicas, que no son excepcionales en esta afección ${ }^{19}$. En ningún caso encontramos los signos motores asimétricos propios de la degeneración corticobasal; y la larga evolución aleja la posibilidad de la variante visual de la enfermedad de Creutzfeldt Jacob ${ }^{20}$.

Las imágenes cerebrales estructurales o funcionales son indispensables en el diagnóstico de ACP. Los exámenes estructurales de rutina pueden ser informados como normales o con atrofia inespecífica, como sucedió en los nuestros, pero estudios más refinados, con volumetría, muestran atrofia de áreas visuales (primarias y de asociación) y de la corteza parietal, mayor a derecha ${ }^{21}$. El compromiso del cingulum posterior fue semejante al de la EA. El estudio de un caso con el compuesto Pittsburgh demostró que el beta amiloide se depositaba preferentemente en la corteza occipital ${ }^{22}$. Nestor y cols estudiaron una serie de pacientes con PET scan, y también encontraron mayor compromiso de la región occípito-parietal, lo que concuerda con el compromiso de la vía visual dorsal, especialmente 
a derecha ${ }^{23}$. Sugiere que el hipometabolismo de los campos oculares frontales, que reciben aferencias de las áreas 18 y 19, podría deberse a la pérdida de esas aferencias y que tendría relación con la apraxia ocular del síndrome de Balint.

Como conclusión general, aceptando que la mayoría de los casos se debería a una EA, debemos señalar que las afecciones neurodegenerativas tienen variantes topográficas: a veces comprometen uno u otro hemisferio, a veces la región

\section{REFERENCIAS}

1. Alberca R. Atrofia cortical posterior. 1999 (www.uninet.edu/union99/congress/ program1. Html, consultado en mayo de 2001).

2. Renner JA, Burns JM, Hou CE, Mckeel DW, Storandt M, MoRRIS JC. Progressive posterior cortical dysfunction: a clinicopathological study. Neurology 2004; 63: $1175-80$.

3. MéndeZ M, Shapira J, Ciark DG. "Apperceptive" alexia in posterior cortical atrophy. Cortex 2007; 43: 264-70.

4. Benson DF, Davis RJ, SNyder BD. Posterior cortical atrophy. Arch Neurol 1988; 45: 789-93.

5. Tang-Wai DF, Grafr-Radford NR, Boeve BF, Dickson DW, PARISI JE, CROOK R ET AL. Clinical, genetic, and neuropathologic characteristics of posterior cortical atrophy. Neurology 2004; 63: 1168-74.

6. McMonagle P, Deering F, Berliner Y, Kertesz A. The cognitive profile of posterior cortical atrophy. Neurology 2006; 66: 331-8.

7. Galton CJ, Patterson K, Xuereb JH, Hodges JR. Atypical and typical presentation of Alzheimer's disease, a clinical, neuropsychological, neuroimaging and pathological study of 13 cases. Brain 2000; 123: 484-98.

8. Aliadi S, Xuereb J, Bak T, Nestor P, Knibb J, Patterson $\mathrm{K}$, Hogdes JR. Focal cortical presentations of Alzheimer's disease. Brain 2007; 130: 2636-45.

9. Victoroff J, Ross GW, Benson DF, Verity MA, Vinters HV. Posterior cortical atrophy. Neuropathologic correlations. Arch Neurol 1994; 51: 269-74.

10. Otsuki M, Soma Y, Tanaka M, Tanaka K, Tanno $Y$, Uesugi Y, TsujI S. [Familial posterior cortical atrophy with visual agnosia and Balint's syndrome] [Article in Japanese] No to Shinkei: 1995; 47: 1185-90. (Resumen consultado en: www.ncbi.nlm.nih.gov/sites/ entrez en marzo de 2008).

11. Donoso A, Manterola JL La atrofia cortical posterior, una variante de la enfermedad de Alzheimer. Rev Neurol Arg 2001; 26: 176-80.

12. RAFAL RD. Balint's syndrome. En: Behavioral neurology and neuropsychology, TE Feinberg y MJ Farah eds, New York, McGraw-Hill Co, 1997. del hipocampo, áreas del lenguaje o áreas prefrontales. En el caso de la ACP el compromiso de las áreas de asociación parietales explicaría los síndromes de Balint y de Gerstmann, el compromiso de las áreas de asociación temporales la existencia de afasias, alexias y agnosias visuales. La causa de estas variaciones no es evidente, podría depender de sutiles diferencias en los procesos patológicos o de la vulnerabilidad selectiva de diversos sistemas neuronales ${ }^{7-9,24}$.

13. Ross SJ, Graham N, Stuart-Green L, Prins M, Xuareb J, Patterson J, Hodges JR. Progressive biparietal atrophy: an atypical presentation of Alzheimer's disease. J Neurol Neurosurg Psychiat 1996; 61: 388-95.

14. Beversdorff DQ, Heilmann KM. Progressive ventral posterior cortical degeneration presenting as alexia for music and words. Neurology 1998; 50: 657-9.

15. Mackenzie S, Graham N, Stuart-Green L Progressive biparietal atrophy: an atipical presentation of Alzheimer's disease. J Neurol Neurosurg Psychiat 1996; 61: 388-95.

16. Bartolomeo P, Dalia Barba G, Boisee MF, BachoudLevi AC, Degos JD, Boler E. Right side neglect in Alzheimer's disease. Neurology 1998; 51: 1207-9.

17. Berthier M, Leiguarda R, Starkstein S, Sevevier G, TARATULO AL. Alzheimer's disease in a patient with posterior cortical atrophy. J Neurol Neurosurg Psychatry 1991; 54: 1110-1.

18. LeVINe DN, LeE JM y FIsCHER CM. The visual variant of Alzheimer's disease: a clinicopathological case study. Neurology 1993; 43: 305-13.

19. Förstl H, Burns A, Levy R, Cairns N, Luthert P, Lantos P. Neurologic signs in Alzheimer's disease. Results of a prospective clinical and neuropathologic study. Arch Neurol 1992; 49: 1038-42.

20. Gálvez S, CarTier L Análisis clínico de una serie de 69 casos definitivos de enfermedad de CreutzfeldtJakob reunidos en Chile entre 1960-1985. Rev Méd Chile 1987; 115: 1148-54.

21. Whitwell JL, Jack CR, Kantarci K, Weigand SD, Boeve BF, KNOPMAN DS ET AL. Imaging correlates of posterior cortical atrophy. Neurobiol Aging 2007; 28: 1051-61.

22. Ng SY, Vimemagne V, Masters C, Rowe CH. Evaluating atypical dementia syndromes using positron emission tomography with carbon 11-labeled Pittsburgh compound B. Arch Neurol 2007; 64: 1140-4.

23. Nestor PJ, Caine D, Fryer TD, Clatke J, Hodges JR. The topography of metabolic deficits in posterior cortical atrophy (the visual variant of Alzheimer's disease) with FDG-PET. J Neurol Neurosurg Psychiat 2003; 74: 1521-9.

24. Donoso A, Behrens MI. Variabilidad y variantes de la enfermedad de Alzheimer. Rev Méd Chile 2005; 133: 477-82. 